\title{
Observation of Mode-Locked Spatial Laser Solitons
}

\author{
F. Gustave ${ }^{1}$ N. Radwell, ${ }^{2}$ C. McIntyre,${ }^{3}$ J. P. Toomey, ${ }^{4}$ D. M. Kane,${ }^{4}$ S. Barland, ${ }^{1}$ \\ W. J. Firth, ${ }^{3}$ G.-L. Oppo, ${ }^{3}$ and T. Ackemann ${ }^{3, *}$ \\ ${ }^{1}$ Université Côte d'Azur, CNRS, 06560 Valbonne, France \\ ${ }^{2}$ SUPA and Department of Physics and Astronomy, University of Glasgow, Glasgow G12 8QQ, United Kingdom \\ ${ }^{3}$ SUPA and Department of Physics, University of Strathclyde, Glasgow G4 ONG, Scotland, United Kingdom \\ ${ }^{4}$ MQ Photonics Research Centre, Department of Physics \& Astronomy, Macquarie University, Sydney 2109, Australia
}

(Received 27 May 2016; published 27 January 2017)

\begin{abstract}
A stable nonlinear wave packet, self-localized in all three dimensions, is an intriguing and much sought after object in nonlinear science in general and in nonlinear photonics in particular. We report on the experimental observation of mode-locked spatial laser solitons in a vertical-cavity surface-emitting laser with frequency-selective feedback from an external cavity. These spontaneously emerging and long-term stable spatiotemporal structures have a pulse length shorter than the cavity round-trip time and may pave the way to completely independent cavity light bullets.
\end{abstract}

DOI: 10.1103/PhysRevLett.118.044102

Wave packets in general, and light wave packets in particular, do not remain confined to small regions in space or time, but have the natural tendency to broaden. In space this is due to diffraction and in time this is due to dispersion, at least in a medium. Although localization can be achieved by confining potentials (e.g., optical fibers and photonic crystals in optics), it was always the vision of researchers to obtain confinement by self-action. This is why the concept of solitary waves, or, more loosely speaking, of solitons, received a lot of attention over the last decades. A soliton is a wave packet in which the tendency to broaden is balanced by nonlinearities. Spatial and temporal solitons in one or two dimensions are known in many fields of science [1-7]. There was also early interest in three-dimensional (3D) self-localization, not least as a model for elementary particles [8]. However, early results [8] were discouraging as they indicated that stationary 3D localized states are not stable in a broad class of nonlinear wave equations. Significant interest continued in the theoretical and mathematical literature (e.g., Refs. [9-15]), but to our knowledge there is only evidence for 3D self-organized periodic patterns [16], but not for 3D self-localization.

In optics, spatiotemporal localization of light, i.e., a "bullet" of light being confined in all three spatial dimensions (and time, because it is propagating), was considered as early as 1990 in the framework of a 3D nonlinear Schrödinger equation (NLSE) [17], but realized to be unstable due to the well-known blow-up experienced for cubic nonlinearities in more than one dimension [18,19]. There were several proposals of how to stabilize multidimensional solitons [19-28]. Spatiotemporal compression [29-31], metastable 2D spatiotemporal solitons [24,32], and metastable discrete light bullets [33,34] were observed in pioneering experiments, the latter constituting the closest realization of a stable light bullet up until now. However, these quasiconservative bullets are only stable over a few characteristic lengths and are long-term unstable due to losses and Raman shifts.

This makes it attractive to look for solitons in dissipative optical systems in which losses are compensated by driving [5]. Indeed solitons can exist in two dimensions with a cubic nonlinearity within a cavity [35] or with parametric driving [12]. Dissipative bullets were analyzed theoretically in the cubic-quintic Ginzburg-Landau model [36-40], intracavity second harmonic generation [41], two-level models for driven cavities [42], optical parametric oscillators [43], solid-state lasers [44] and semiconductor lasers [45]. In particular, the latter seem to be very promising as advances in semiconductor laser technology yielded 2D spatial solitons $[4,46-48]$ as well as 1D temporal solitons and mode locking [49-52]. The latter matches a huge literature on temporal dissipative solitons in mode-locked solid-state and fiber lasers, see, e.g., Ref. [3]. Hence, it seems to be attractive to look at mode-locking spatial laser solitons to achieve 3D localization.

We are addressing the issue in a vertical-cavity surfaceemitting laser (VCSEL) with frequency-selective feedback (FSF) from an external cavity [46,53], and demonstrate self-pulsing of spatial solitons based on the locking of three external cavity modes. This constitutes already an important novel experimental observation in optical self-organization and nonlinear photonics. Furthermore, these spatiotemporal structures result from self-organization on scales shorter than the spatial extent of the compound cavity in all three dimensions of space and, therefore, pave the way to light bullets.

The experimental system [Fig. 1(a)] consists of a VCSEL with an InGaAs gain material emitting around $980 \mathrm{~nm}$ [53] and an aperture diameter of $200 \mu \mathrm{m}$. The laser 
(a)

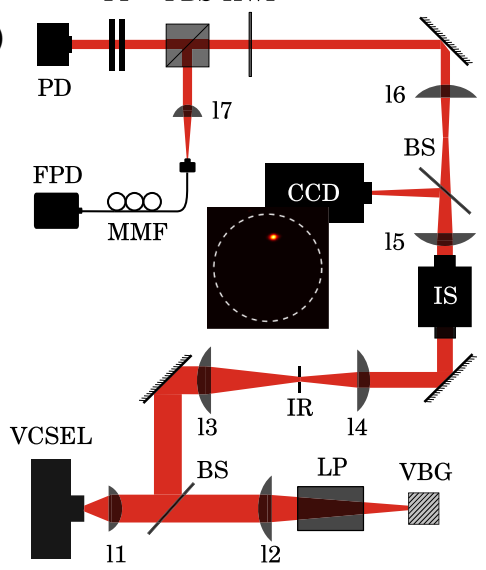

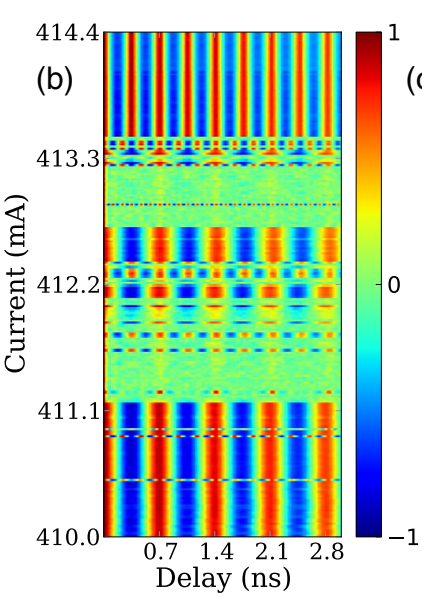

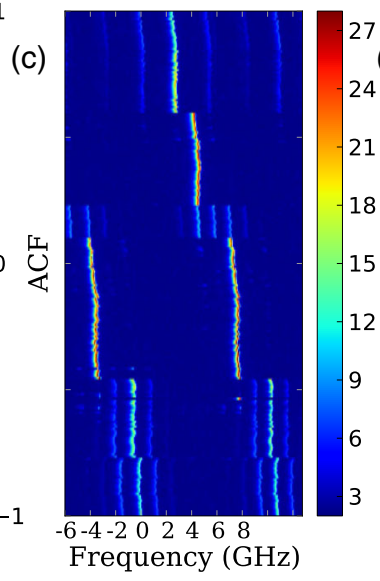

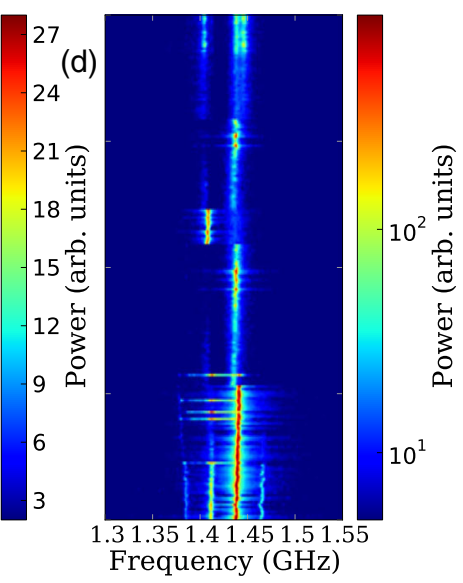

FIG. 1. (a) Scheme of experimental setup. The inset illustrates spatial localization by showing the soliton within the much larger pumped VCSEL aperture indicated by the dotted white line. Lens (L), beam sampler (BS), linear polarizer (LP), volume Bragg grating (VBG), iris (IR), optical isolator (IS), charge-coupled device (CCD) camera, half-wave plate (HWP), polarizing beam splitter (PBS), Fabry-Perot (FP) interferometer [free spectral range (FSR) of $12 \mathrm{GHz}$ and a Finesse of about 40], photodector (PD), fast photodetector (FPD). (b)-(d) Correlation functions and spectra obtained for different currents (scanning down) in the bistable range, (b) Autocorrelation function, (c) Optical spectra obtained from FP (the overall blueshift with decreasing current is due to the reduced thermal load; approximately 1.7 FSR are displayed to show unwrapped spectra in spite of the blueshift) and (d) rf spectra of the intensity signal on the FPD from an electrical spectrum analyzer (ESA) around the fundamental round-trip frequency.

is coupled to a self-imaging external cavity closed by a volume Bragg grating (VBG) as a frequency filter. The VBG has a reflectivity peak with $99 \%$ reflection at around $981 \mathrm{~nm}$ with a full width at half maximum (FWHM) of $30 \mathrm{GHz}$. The round-trip time in the external cavity is about 0.7 ns. The output is monitored locally with a fast photodetector (bandwidth $9.5 \mathrm{GHz}$ ) followed by an accoupled rf amplifier (bandwidth $15 \mathrm{GHz}$ ) and is then digitized by a real time oscilloscope at $100 \mathrm{GS} / \mathrm{s}$ (bandwidth $23 \mathrm{GHz}$ ). A linear polarizer forces the polarization of the solitons to be vertical ensuring $10 \%$ outcoupling via the Fresnel reflection of an intracavity beam sampler. The detected area is broader than the soliton; i.e., the detector is only sensitive to variations of total power and not to breathing or jittering modes. The optical spectrum is monitored with a plano-planar scanning Fabry-Perot interferometer. Changing the current does not only change the gain, but the dominant effect is actually to change the detuning between VCSEL resonance and VBG resonance via Ohmic heating (e.g., Refs. [54,55]). This change of detuning is our main control parameter. For small enough detuning, optical bistability occurs due to a carrier mediated, dispersive nonlinearity [54-56]. Situations of singlemode operation in the external cavity (corresponding to $\mathrm{cw}$ spatial solitons [53]) and multimode operation can be realized. Some self-pulsing was found in preliminary investigations $[55,62,63]$. The measurements reported in this Letter demonstrate long-term mode locking of solitons with an excellent phase coherence. We stress that the modelocked states reported here are bistable [56]; i.e., it can be either present or absent for fixed values of the parameters, a typical feature of localized dissipative structures.
In the realization discussed here (VCSEL substrate temperature $70.5^{\circ} \mathrm{C}$ ), the soliton switches on at $414.4 \mathrm{~mA}$, as the current is ramped up slowly in steps of $0.02 \mathrm{~mA}$. After a value of $418 \mathrm{~mA}$ is reached, the current is ramped down again and the soliton is sustained below the switch-on threshold to a switch-off threshold below $409.9 \mathrm{~mA}$. In the current range investigated in Figs. 1(b)-1(d), the system is bistable; i.e., the soliton coexists with the nonlasing state as expected for a localized dissipative structure. Depending on current, the autocorrelation function [ACF, Fig. 1(b)] shows distinct recurrences at multiples and fractions of the cavity round-trip time of $0.7 \mathrm{~ns}$ as well as very unstructured regimes. Comparison with the optical spectra in Fig. 1(c) shows that regular recurrence is typically associated with multimode dynamics providing a first indication of selfpulsing via mode locking. This is supported by the radiofrequency (rf) spectra [Fig. 1(d)], showing a pronounced peak at the cavity round-trip frequency around $1.4 \mathrm{GHz}$ for the regions of regular ACF recurrence at 410-411.1, 412.1, and around $412.5 \mathrm{~mA}$. We are going to discuss typical examples for three different manifestations of regular selfpulsing spatial solitons below.

Figure 2 depicts the situation at a current of $413.7 \mathrm{~mA}$. A fairly regular sequence of pulses is emitted with a pulse separation of $351 \mathrm{ps}$ (standard deviation (SDV) $10 \mathrm{ps).} \mathrm{The}$ dominant rf feature is found just below $2.85 \mathrm{GHz}$. In the terminology of mode locking, this corresponds to harmonic mode locking, i.e., at 2 times the fundamental round-trip frequency. Indeed there are two pulses within the cavity at the same time as demonstrated by the space-time representation in the right panel of Fig. 2. The horizontal axis displays time within a round-trip frame (spacelike, [64]) 

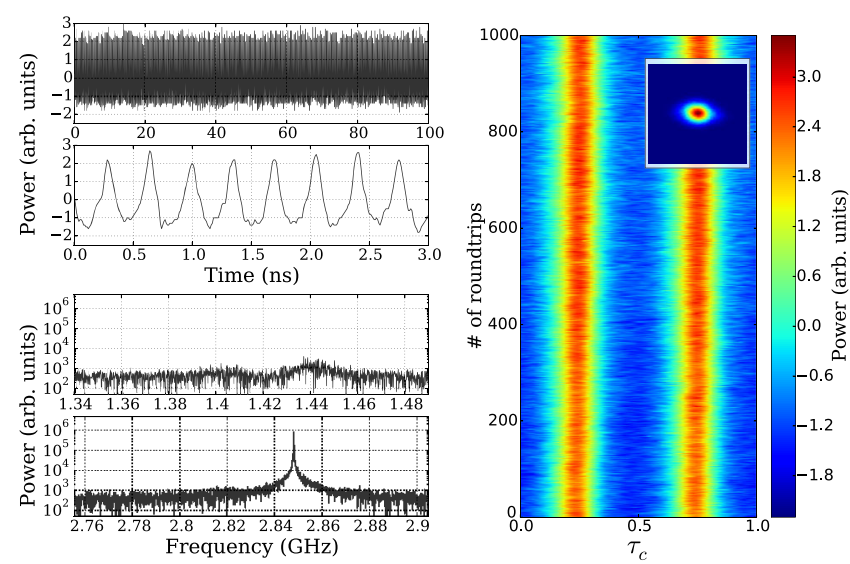

FIG. 2. Example of harmonic mode locking, $I=413.7 \mathrm{~mA}$. Left panel, from top to bottom: Envelope of time series, zoom on pulses (the ac-coupled rf amplifier removes the dc component and hence the signal has positive and negative amplitudes), rf spectra obtained from a numerical fast Fourier transform (FFT) of the recorded time series around the fundamental round-trip frequency, rf spectrum around harmonic. The right panel shows space-time representations of the dynamics, the horizontal axis displays time within a round-trip (spacelike) and the vertical axis the number of round-trips. The inset shows the time averaged intensity profile of the pulsing soliton in a square with a size of $55 \mu \mathrm{m}$.

and the vertical axis displays slow time developing with the number of round-trips. The optical spectrum in Fig. 1(c) confirms that the excited modes are separated by two FSR of the external cavity. There are three modes excited with two further faint side modes. The average pulse width (FWHM) measured in the time domain is 112 ps (SDV $9 \mathrm{ps}$ ). This is in agreement with a numerical estimation of 116 ps obtained from the locking of three modes with the relative amplitudes as observed in the optical spectrum [56]. The width of the rf peak is at the resolution limit given by the observation time of $1 / 40 \mu \mathrm{s}=25 \mathrm{kHz}$. This evidences excellent phase synchronization. The envelope is also fairly stable over long time scales. The observations provide evidence of robust harmonic mode locking of spatial solitons. We stress that the mode locking prevails over much longer scales (seconds to minutes) than the recorded time span of $40 \mu \mathrm{s}$, which corresponds already to about 57000 round-trips. Its stability is limited by drifts in the setup changing the detuning conditions, as there is no active stabilization of cavity length.

Figure 3 illustrates the situation at a lower current of $410.6 \mathrm{~mA}$. Again, there is a fairly stable sequence of pulses, but the pulse separation is now $695 \mathrm{ps}$ (SDV $31 \mathrm{ps)} \mathrm{and} \mathrm{the}$ fundamental repetition frequency is at $1.438 \mathrm{GHz}$. The optical spectrum in Fig. 1(c) shows again three main modes with two much fainter sidemodes. They are separated by only one FSR of the external cavity. The measured pulse duration is 270 (SDV 23 ps). This is again in good agreement with an estimation of $250 \mathrm{ps}$ stemming from
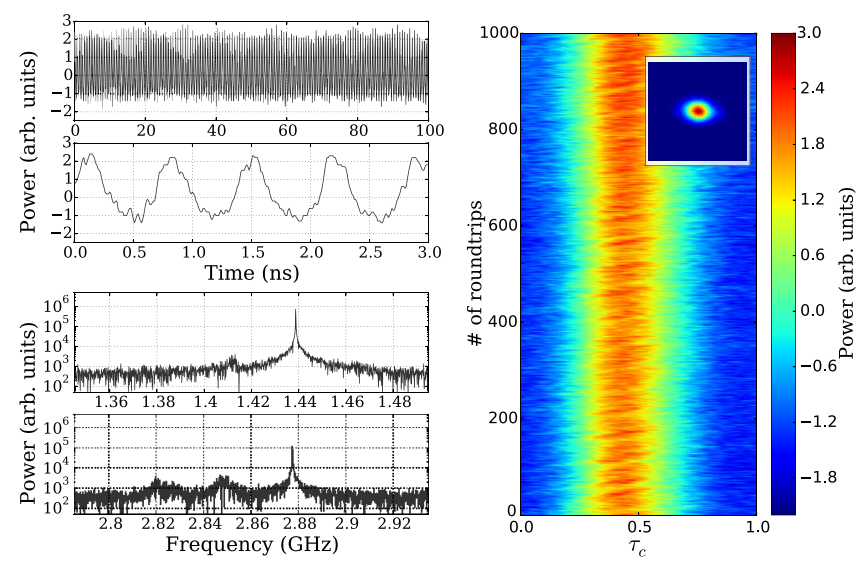

FIG. 3. Example of fundamental mode locking, $I=410.6 \mathrm{~mA}$. Explanation of subpanels as in Fig. 2.

a numerical superposition of three modes [56]. As the rf amplifier in the experiment is ac coupled, we do not have direct access to the modulation depth, but the numerical superposition of three modes indicates an effective valleypulse peak modulation depth of $91 \%-94 \%$, and thus provides indirect confirmation of a high modulation depth also in experiment [56]. There is only one pulse per roundtrip. This constitutes fundamental mode locking. The fundamental rf peak is still resolution limited, confirming excellent phase correlation. Again, the envelope is fairly stable. There are weak indications of satellite peaks around the fundamental rf peak but they are suppressed by nearly 3 orders of magnitude.

The sidebands are undamped for lower currents (e.g., $410.24 \mathrm{~mA}$, Fig. 4) leading to a regular modulation of the envelope at low frequencies ( $27 \mathrm{MHz}$ ) as evident from the time series and the space-time plot. A corresponding peak exists in the low-frequency rf spectrum. The modulation frequency also appears as a difference frequency between the sidebands in the rf spectrum around the round-trip frequency where it is important to note that each of them remains very narrow $(<100 \mathrm{kHz} \quad \mathrm{FWHM})$. This
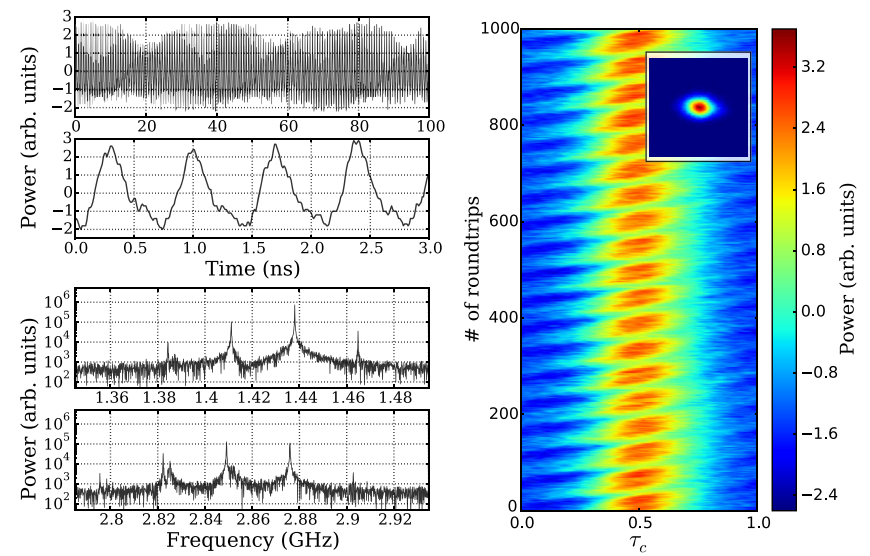

FIG. 4. Example of quasiperiodic dynamics in mode locking, $I=410.24 \mathrm{~mA}$. Explanation of subpanels as in Fig. 2. 
corresponds to quasiperiodic motion. Corresponding instabilities of the envelope of a mode-locked pulse train are known for Ti:sapphire and fiber lasers $[65,66]$ and can be described by the cubic-quintic Ginzburg-Landau equation $[66,67]$.

The numerical integration of an established model for the dynamics of a VCSEL with FSF [68] (see the Supplemental Material [56]) confirms the possibility of mode-locked dynamics of spatial solitons. The parameters used are best estimations for the VCSEL under study and were used before to describe $\mathrm{cw}$ solitons [68] and transient pulsing dynamics [69]. Stable mode locking was found for cavity round-trip times below $0.7 \mathrm{~ns}$ with the detuning $\theta$ between the VCSEL and the VBG being a sensitive parameter. An example of mode-locked dynamics obtained for a cavity round-trip time of $0.28 \mathrm{~ns}$ is displayed in Fig. 5, showing a pulsing structure very similar to the experimental one. Highly regular pulses are emitted close to the harmonic round-trip frequency. The widths of the first rf peaks are resolution limited, confirming high quality phase correlation. The FWHM of the pulses is about $40 \mathrm{ps}$, the modulation depth $78 \%$. The numerical analysis also confirms that the pulsing is due to an amplitude modulation of the whole profile of the soliton, not a breathing or motional mode. Cavities with delay times above $0.7 \mathrm{~ns}$ yielded irregular dynamics. In the experiment, stable mode locking disappeared in longer cavities (1.05 ns round-trip time) in favor of irregular dynamics, too. This constitutes a remarkable qualitative agreement. The large parameter space and the high computational load prevent a more exhaustive analysis of the interplay of simulation parameters like $\alpha$-factor, delay time and carrier lifetime in stable mode locking at the present stage.

From the observations, we infer mode-locked dynamics of spatial laser solitons. As the width of the temporal
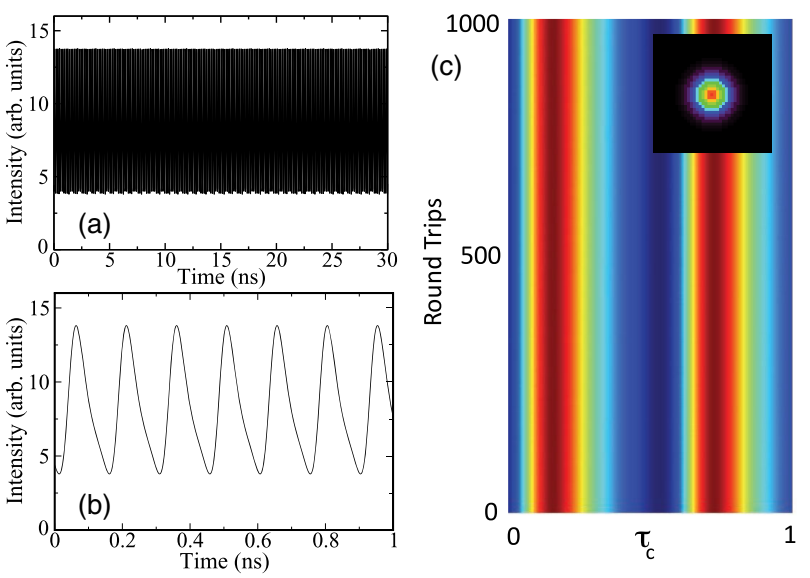

FIG. 5. Numerical results on mode-locked soliton for a cavity round-trip time of $0.28 \mathrm{~ns}$. (a) Envelope of time series, (b) zoom on pulses, (c) space-time representation. Parameters (see Ref. [68]): $\alpha=9, \theta=-0.95, \sigma=0.9, \gamma=0.01, T_{1}=0.008$, $T_{2}=0.002, \beta=0.6, r_{g}=0.8, \delta=0, \tau_{f}=28$. The inset shows a snapshot of the intensity profile of the pulsing soliton. structure is shorter than the cavity round-trip time, this provides evidence for a temporal bullet in the cavity, or, more carefully, as the modulation is not complete, of a bullet component in the dynamics. A 3D bullet reconstructs itself 2 times within the round-trip time (4 times for harmonic mode locking), in the VCSEL and at the VBG. This is in line with the fact that, unless the resonator is completely filled with the nonlinear material, the spatial soliton is only well localized in a few planes within the cavity $[46,47,70,71]$. Similarly, large variations of pulse parameters along the cavity might occur for temporal dissipative cavity solitons in lasers that contain discrete gain, loss, and positive and negative dispersion elements [3].

Self-pulsing instabilities in semiconductor lasers are usually discussed in the framework of the excitation of relaxation oscillations (RO) interacting with the pulsing at the round-trip time [72,73]. For cavities with a round-trip time shorter than the inverse of the RO frequency, fairly regular dynamics in the form of so-called regular pulse packages is possible [74-76], but the packages are much shorter than observed here and, more importantly, no stableself pulsing with a constant envelope was reported in the literature. We do not observe the RO frequency in the output from the VCSEL and the expectation would be that it would exceed the $1.4 \mathrm{GHz}$ external cavity frequency, and thus we do not expect the RO to be the dominant driving mechanism.

Looking for the nonlinear mechanisms destabilizing the cw solution, it was realized already some time ago [77,78] that a mechanism for nonlinear loss saturation providing passive mode locking [3], might be at work in semiconductor lasers with FSF due to the strong phaseamplitude coupling [79]. A reduction of carrier density leads to an increase in refractive index which shifts the resonance of the VCSEL further into resonance with the VBG thus reducing losses. This loss reduction can overcompensate the gain reduction and it was argued to provide a mechanism to amplify fluctuations and multilongitudinal mode operation. The possibility of regular, slightly anharmonic oscillations with FSF was noticed [77]. Four wave mixing mediated by phase-amplitude coupling will also contribute to phase locking as noted before for semiconductor lasers without a saturable absorber section [80,81], soliton formation in dielectric microcavities [82], and dissipative parametric instabilities [83]. Mechanisms limiting the pulse width are gain dispersion due to the filter bandwidth and the group velocity dispersion introduced by the narrow VCSEL resonance. For the parameters of Fig. 5 this can be estimated to be about $-80 \mathrm{ps}^{2}$ for the main optical mode taking the effective cavity resonance at the carrier density in the minimum of the pulse as reference to measure detuning. This means that most of the optical spectrum is in the anomalous dispersion regime, i.e., in temporal soliton territory, albeit with strong high order dispersion. Note that normal group velocity dispersion would not 
necessarily exclude the stability of light bullets due to space-time coupling [39] and that the existence range of multidimensional solitons is typically enhanced in dissipative schemes as argued in the introduction.

Having established a possible mechanism for mode locking and nonlinear pulse shaping making these structure dissipative solitons in the terminology of Ref. [3], there is the question whether they are truly self-localized states in the sense that they are independent from each other. This question was recently addressed for temporal solitons in a VCSEL with saturable absorber [51] and a difference between the harmonically mode-locked state, in which solitons are locked to a rigid temporal pattern at maximum distance by the need for gain recovery, and independent localized structures with, in principle, arbitrary positioning was established. We do not have the equipment for addressing pulses at the subcavity round-trip time scale but inspection of Fig. 2 indicates that there might be some jitter of pulse to pulse separations. A quantitative analysis of the SDV of the pulse separations yields a slightly larger jitter between adjacent pulses than between pulses separated by the round-trip time [56]. One needs to be careful as the tendency is at the edge of statistical significance, but these observations open the tantalizing prospect that the pulses not only represent mode-locked spatial solitons but pave the way to truly mutually independent cavity light bullets.

We are grateful to Roland Jäger (Ulm Photonics) for supplying the devices, Jesus Jimenez and Paul Griffin for help with the experimental setup, Jiazhu Pan for advice on the analysis of the uncertainty of the jitter data, Svetlana Gurevich for literature on reaction diffusion systems, and LeCroy and Tektronix for lending us marvelous fast digitizing oscilloscopes. N.R. and G. McI gratefully acknowledge financial support from the EPSRC. The Macquarie group gratefully acknowledges support by a Science and Industry Endowment Fund (SIEF) grant. The collaboration between the British and Australian group was supported by the Royal Society (London), and between the British and the French group by the Global Engagement Fund of the University of Strathclyde and the Agence Nationale de la Recherche through Grant No. ANR-12JS04-0002-01.

*Corresponding author. thorsten.ackemann@strath.ac.uk

[1] M. Remoissenet, Waves Called Solitons: Concepts, Experiments (Springer, Berlin, Heidelberg, New York, 1994).

[2] G. I. Stegeman and M. Segev, Science 286, 1518 (1999).

[3] P. Grelu and N. Akhmediev, Nat. Photonics 6, 84 (2012).

[4] T. Ackemann, G.-L. Oppo, and W. J. Firth, Adv. At. Mol. Opt. Phys. 57, 323 (2009).

[5] N. Akhmediev and A. Ankiewicz, Dissipative Solitons, Lecture Notes in Physics (Springer, Berlin, 2005), Vol. 661.
[6] N. Akhmediev and A. Ankiewicz, Dissipative Solitons: From Optics to Biology and Medicine, Lecture Notes in Physics (Springer, New York, 2008), Vol. 751.

[7] H. G. Purwins, H. U. Bödeker, and S. Amiranashvili, Adv. Phys. 59, 485 (2010).

[8] G. H. Derrick, J. Math. Phys. (N.Y.) 5, 1252 (1964).

[9] C. B. Muratov and V. V. Osipov, Phys. Rev. E 53, 3101 (1996).

[10] C. B. Muratov and V. V. Osipov, J. Phys. A 33, 8893 (2000).

[11] M. Bode, A. Liehr, C. Schenk, and H. G. Purwins, Physica (Amsterdam) 161D, 45 (2002).

[12] I. V. Barashenkov, N. V. Alexeeva, and E. V. Zemlyanaya, Phys. Rev. Lett. 89, 104101 (2002).

[13] N. Graham, Phys. Rev. Lett. 98, 101801 (2007).

[14] M. A. Amin, R. Easther, and H. Finkel, J. Cosmol. Astropart. Phys. 12 (2010) 001.

[15] U. Thiele, A. J. Archer, M. J. Robbins, H. Gomez, and E. Knobloch, Phys. Rev. E 87, 042915 (2013).

[16] T. Bánsági Jr., V. K. Vanag, and I. R. Epstein, Science 331, 1309 (2011).

[17] Y. Silberberg, Opt. Lett. 15, 1282 (1990).

[18] J. J. Rasmussen and K. Rypdal, Phys. Scr. 33, 481 (1986).

[19] L. Bergé, Phys. Rep. 303, 259 (1998).

[20] A. A. Kolokolov, J. Appl. Mech. Tech. Phys. 14, 426 (1973).

[21] R. H. Enns and S. S. Rangnekar, Phys. Rev. A 45, 3354 (1992).

[22] N. Akhmediev and J. M. Soto-Crespo, Phys. Rev. A 47, 1358 (1993).

[23] N. A. Veretenov, A. G. Vladimirov, N. A. Kaliteevski, N. N. Rozanov, S. V. Fedorov, and A. N. Shatsev, Opt. Spectrosc. 89, 380 (2000).

[24] F. W. Wise and P. Di Trapani, Opt. Photonics News 13, 28 (2002).

[25] D. Mihalache, D. Mazilu, F. Lederer, Y. V. Kartashov, L.-C. Crasovan, and L. Torner, Phys. Rev. E 70, 055603(R) (2004).

[26] B. A. Malomed, D. Mihalache, F. Wise, and L. Torner, J. Opt. B 7, R53 (2005).

[27] P. Panagiotopoulos, O. Whalen, M. Kolesik, and J. V. Moloney, Nat. Photonics 9, 543 (2015).

[28] N. A. Veretenov, N. N. Rosanov, and S. V. Fedorov, Phys. Rev. Lett. 117, 183901 (2016).

[29] I. G. Koprinkov, A. Suda, P. Wang, and K. Midorikawa, Phys. Rev. Lett. 84, 3847 (2000).

[30] A. L. Gaeta and F. Wise, Phys. Rev. Lett. 87, 229401 (2001).

[31] H. S. Eisenberg, R. Morandotti, Y. Silberberg, S. Bar-Ad, D. Ross, and J. S. Aitchison, Phys. Rev. Lett. 87, 043902 (2001).

[32] X. Liu, L. J. Qian, and F. W. Wise, Phys. Rev. Lett. 82, 4631 (1999).

[33] S. Minardi, F. Eilenberger, Y. V. Kartashov, A. Szameit, U. Röpke, J. Kobelke, K. Schuster, H. Bartelt, S. Nolte, L. Torner, F. Lederer, A. Tünnermann, and T. Pertsch, Phys. Rev. Lett. 105, 263901 (2010).

[34] F. Eilenberger, K. Prater, S. Minardi, R. Geiss, U. Röpke, J. Kobelke, K. Schuster, H. Bartelt, S. Nolte, A. Tünnermann, and T. Pertsch, Phys. Rev. X 3, 041031 (2013).

[35] W. J. Firth and A. Lord, J. Mod. Opt. 43, 1071 (1996). 
[36] P. Grelu, J. M. Soto-Crespo, and N. Akhmediev, Opt. Express 13, 9352 (2005).

[37] V. Skarka and N. B. Aleksic, Phys. Rev. Lett. 96, 013903 (2006).

[38] D. Mihalache, D. Mazilu, F. Lederer, Y. V. Kartashov, L.-C. Crasovan, L. Torner, and B. A. Malomed, Phys. Rev. Lett. 97, 073904 (2006).

[39] N. N. Akhmediev, J. M. Soto-Crespo, and P. Grelu, Chaos 17, 037112 (2007).

[40] D. Mihalache, D. Mazilu, F. Lederer, H. Leblond, and B. A. Malomed, Phys. Rev. A 75, 033811 (2007).

[41] M. Tlidi and P. Mandel, Phys. Rev. Lett. 83, 4995 (1999).

[42] M. Brambilla, T. Maggipinto, G. Patera, and L. Columbo, Phys. Rev. Lett. 93, 203901 (2004).

[43] N. Veretenov and M. Tlidi, Phys. Rev. A 80, 023822 (2009).

[44] W. H. Renninger and F. W. Wise, Optica 1, 101 (2014).

[45] J. Javaloyes, Phys. Rev. Lett. 116, 043901 (2016).

[46] Y. Tanguy, T. Ackemann, W. J. Firth, and R. Jäger, Phys. Rev. Lett. 100, 013907 (2008).

[47] P. Genevet, S. Barland, M. Giudici, and J. R. Tredicce, Phys. Rev. Lett. 101, 123905 (2008).

[48] T. Elsass, K. Gauthron, G. Beaudoin, I. Sagnes, R. Kuszelewicz, and S. Barbay, Eur. Phys. J. D 59, 91 (2010).

[49] R. Paschotta, R. Häring, A. Garnache, S. Hoogland, A. C. Tropper, and U. Keller, Appl. Phys. B 75, 445 (2002).

[50] P. Genevet, S. Barland, M. Giudici, and J. R. Tredicce, Phys. Rev. A 79, 033819 (2009).

[51] M. Marconi, J. Javaloyes, S. Balle, and M. Giudici, Phys. Rev. Lett. 112, 223901 (2014).

[52] F. Gustave, L. Columbo, G. Tissoni, M. Brambilla, F. Prati, B. Kelleher, B. Tykalewicz, and S. Barland, Phys. Rev. Lett. 115, 043902 (2015).

[53] N. Radwell and T. Ackemann, IEEE J. Quantum Electron. 45, 1388 (2009).

[54] J. Jimenez, G.-L. Oppo, and T. Ackemann, J. Phys. D 49, 095110 (2016).

[55] T. Ackemann, J. Jimenez, Y. Noblet, N. Radwell, G. Ren, P. Paulau, C. McIntyre, G.-L. Oppo, J. P. Toomey, and D. M. Kane, Dynamics and interaction of laser cavity solitons in broad-area semiconductor lasers, in Nonlinear Optical Cavity Dynamics, edited by P. Grelu (Wiley, New York, 2016), Chap. 3, p. 41.

[56] See Supplemental Material at http://link.aps.org/ supplemental/10.1103/PhysRevLett.118.044102 for details on bistability of solitons, numerical reconstruction of modelocked time series from three modes, and the statistical analysis of pulse jitter, which includes Refs. [57-61].

[57] L. A. Lugiato, Prog. Opt. XXI, 69 (1984).

[58] L. Spinelli, G. Tissoni, M. Brambilla, F. Prati, and L. A. Lugiato, Phys. Rev. A 58, 2542 (1998).

[59] R. Lang and K. Kobayashi, IEEE J. Quantum Electron. 16, 347 (1980).
[60] M. Giudici, S. Balle, T. Ackemann, S. Barland, and J. R. Tredicce, J. Opt. Soc. Am. B 16, 2114 (1999).

[61] C. McIntyre, Ph.D. thesis, SUPA and Department of Physics, University of Strathclyde, 2013.

[62] T. Ackemann, N. Radwell, C. McIntyre, G.-L. Oppo, and W. J. Firth, in 12th International Conference on Transparent Optical Networks (ICTON) (IEEE, Bellingham, WA, 2010), p. Th.B1.1.

[63] J. P. Toomey, D. M. Kane, and T. Ackemann, Opt. Express 22, 17840 (2014).

[64] G. Giacomelli and A. Politi, Phys. Rev. Lett. 76, 2686 (1996).

[65] S. R. Bolton and M. R. Acton, Phys. Rev. A 62, 063803 (2000).

[66] J. M. Soto-Crespo, M. Grapinet, P. Grelu, and N. Akhmediev, Phys. Rev. E 70, 066612 (2004).

[67] N. N. Akhmediev, J. M. Soto-Crespo, and G. Town, Phys. Rev. E 63, 056602 (2001).

[68] A. J. Scroggie, W. J. Firth, and G.-L. Oppo, Phys. Rev. A 80, 013829 (2009).

[69] N. Radwell, C. McIntyre, A. J. Scroggie, G.-L. Oppo, W. J. Firth, and T. Ackemann, Eur. Phys. J. D 59, 121 (2010).

[70] V. B. Taranenko, K. Staliunas, and C. O. Weiss, Phys. Rev. A 56, 1582 (1997).

[71] M. Saffman, D. Montgomery, and D.Z. Anderson, Opt. Lett. 19, 518 (1994).

[72] J. Mørk, J. Mark, and B. Tromborg, Phys. Rev. Lett. 65, 1999 (1990).

[73] K. Petermann, IEEE J. Sel. Top. Quantum Electron. 1, 480 (1995).

[74] T. Heil, I. Fischer, W. Elsäßer, and A. Gavrielides, Phys. Rev. Lett. 87, 243901 (2001).

[75] A. Tabaka, K. Panajotov, I. Veretennicoff, and M. Sciamanna, Phys. Rev. E 70, 036211 (2004).

[76] J. P. Toomey, D. M. Kane, C. McMahon, A. Argyris, and D. Syvridis, Opt. Express 23, 18754 (2015).

[77] P. Zorabedian, IEEE J. Quantum Electron. 30, 1542 (1994).

[78] W. R. Trutna, Jr. and P. Zorabedian, Hewlett-Packard Journal 44, 35 (1993).

[79] C. H. Henry, IEEE J. Quantum Electron. 18, 259 (1982).

[80] J. Renaudier, G.-H. Duan, J.-G. Provost, H. DebregeasSillard, and P. Gallion, IEEE Photonics Technol. Lett. 17, 741 (2005).

[81] C. Gosset, K. Merghem, A. Martinez, G. Moreau, G. Patriarche, G. Aubin, and A. Ramdane, Appl. Phys. Lett. 88, 241105 (2006).

[82] T. Herr, V. Brasch, J. D. Jost, C. Y. Wang, N. M. Kondratiev, M. L. Gorodetsky, and T. J. Kippenberg, Nat. Photonics 8, 145 (2014).

[83] A. M. Perego, N. Tarasov, D. V. Churkin, S. K. Turitsyn, and K. Staliunas, Phys. Rev. Lett. 116, 028701 (2016). 